\title{
ESTRESORES DOCENTES EN TIEMPOS DE PANDEMIA: UN INSTRUMENTO PARA SU EXPLORACIÓN
}

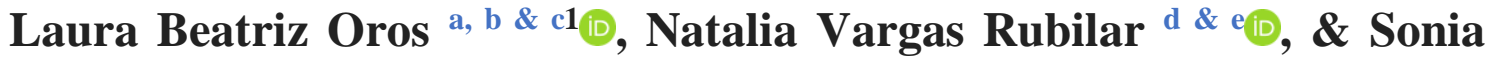 \\ Chemisquy ${ }^{\mathfrak{f}\left(\mathbb{C}^{2}\right.}{ }^{2}$
}

Universidad Católica Argentina, Buenos Aires, Argentina a, Consejo Nacional de Investigaciones Científicas y Técnica, Buenos Aires, Argentina b; Universidad Adventista del Plata, Libertador San Martín, Argentina c; Instituto de Educación Superior N9-027, Guaymallén, Argentina $^{\mathrm{d}}$; Universidad del Aconcagua, Mendoza, Argentina ${ }^{\mathrm{e}}$; Centro de Asistencia y Formación en Psicopedagogía, Posadas, Misiones ${ }^{\mathrm{f}}$.

\section{RESUMEN}

Los profesionales de la educación representan uno de los colectivos ocupacionales con mayor riesgo de desarrollar estrés y desgaste profesional, situación que puede haberse amplificado a partir de los cambios laborales acontecidos en el marco de la pandemia por COVID-19. El objetivo de este trabajo, de corte instrumental, fue desarrollar y validar una escala para evaluar la percepción de estresores docentes en contexto de aislamiento social. Participaron de forma anónima y voluntaria 674 docentes argentinos $\left(M_{E D A D}\right.$ $=39.08 ; D E=9.10 ; 72.2 \%$ mujeres, $22.8 \%$ hombres) quienes completaron una versión inicial de la escala con 23 ítems. Mediante análisis factoriales exploratorios y confirmatorios, se retuvieron 21 ítems que evalúan cinco factores estresantes: el entorno de trabajo y la sobrecarga laboral, el empleo de nuevas tecnologías, la incertidumbre por la duración y consecuencias de la pandemia, el aspecto organizacional de la institución educativa, y las relaciones con el entorno del alumno, el conflicto y la ambigüedad de rol. La consistencia interna de la escala completa resultó excelente $(\omega=.95)$. Afín con la teoría, y como evidencia de validez nomológica, se observó una correlación positiva entre la percepción de estresores y los síntomas psicofísicos de estrés $(\mathrm{r}=.54 ; \mathrm{p}<.001)$.

Palabras Claves

estrés ocupacional; docentes; cuestionario; pandemias; aislamiento social

\begin{abstract}
Teachers represent one of the most endangered professional groups for the development of stress and burnout, risks that could have gotten enhanced due to work changes related to the COVID-19 pandemic. The aim of this work was to develop and validate a measure to assess teachers' perceptions of stressors in the context of social distancing. The sample was composed by 674 anonymous and volunteer argentine teachers (MAGE $=39.08 ; \mathrm{SD}=9.10 ; 72.2 \%$ female, $22.8 \%$ male) who completed an initial version of the scale, integrated by 23 items. After exploratory and confirmatory factor analyses, 21 items were retained, representing five stress factors: work environment and work overload, the use of new technologies, the uncertainty regarding the duration and consequences of the pandemics, the organizational aspects of the educational institution, and the relationships with the student's environment, the conflict and role ambiguity. The internal consistency of the complete scale was excellent $(\omega=.95)$. In line with the theory, and as evidence of nomological validity, the correlation obtained with a scale of psychophysics symptoms of stress was positive $(\mathrm{r}=.53 ; \mathrm{p}=.000)$.
\end{abstract}

Keywords

work-related stress; teachers; scale; pandemics; social distancing

\footnotetext{
1 Correspondence about this article should be addressed to Laura Beatriz Oros: 1auraorosb@gmail.com

2 Conflicts of Interest: The authors declare that the research was conducted in the absence of any commercial or financial relationships that could be construed as a potential conflict of interest.
} 


\section{TEACHERS' STRESSORS IN TIMES OF PANDEMIC: AN INSTRUMENT FOR THEIR EXPLORATION}

\section{Introducción}

El 11 de marzo de 2020 la Organización Mundial de la Salud declaró al brote del nuevo coronavirus (COVID-19) como una pandemia. Frente a esta situación, el 20 de marzo del presente año el Poder Ejecutivo de la Nación Argentina dispuso el aislamiento social, preventivo y obligatorio como una medida para prevenir los contagios y mitigar la circulación del virus. A partir de ese momento, las instituciones educativas de todo el país debieron reorganizarse para garantizar la continuidad del ciclo lectivo de manera no presencial. Transcurridas las primeras semanas de la cuarentena obligatoria, algunos sondeos periodísticos realizados en diferentes provincias argentinas indicaron que bajo estas circunstancias muchos docentes manifestaron sentirse sobrepasados por el cambio vertiginoso que implicaba educar a distancia, comenzando a evidenciarse así un deterioro en la salud física y emocional de los profesionales de la educación (Carnese, 2020; Di Vincenzo, 2020; Favant, 2020; Fernández, 2020; Figueroa Díaz, 2020; Santoro, 2020; Vallejos, 2020). Esta situación justifica la pronta realización de investigaciones psicológicas con rigor científico que permitan identificar los desencadenantes, las características y las consecuencias del estrés laboral docente, a fin de tomar medidas que garanticen que en estos tiempos complejos los procesos de enseñanza y de aprendizaje se realicen de la manera más eficiente que sea posible, implicando a la vez el menor costo de riesgo psicofísico para los educadores.

A lo largo de los años, numerosos estudios han señalado a los profesionales de la educación como uno de los colectivos ocupacionales que presentan mayor riesgo de desarrollar estrés, ansiedad y el síndrome de quemarse por el trabajo (Gil-Monte, 2005; Gil-Monte \& Peiró, 1997; Manassero et al., 2003; Othman \& Sivasubramaniam, 2019; Schaufeli, 2003; Schaufeli \& Enzmann, 1998; Vandenberghe \& Huberman, 1999). Fundamentalmente, porque estos profesionales se encuentran expuestos a un amplio abanico de estresores laborales en su quehacer diario (Esteve, 1994; Menghi, 2015).

Durante los últimos meses los docentes han visto modificados varios aspectos de su trabajo; por ejemplo, el lugar donde desarrollan su tarea laboral, el tiempo y los recursos que emplean para enseñar y los canales de comunicación. Posiblemente, estos cambios acontecidos en el entorno laboral y en las condiciones de trabajo han conllevado 
un incremento en la percepción de estresores laborales y/o en la intensidad atribuida a los mismos por parte de los educadores.

De acuerdo a la teoría transaccional propuesta por Lazarus y Folkman (1986), el estrés surge como resultado de una relación particular entre la persona y el entorno; específicamente, cuando la persona percibe que las demandas del medio amenazan su bienestar y desbordan su capacidad de respuesta. La percepción de la amenaza se refiere a la consideración que hace el individuo, por medio de un proceso de valoración cognitiva e idiosincrática, de los estímulos externos que lo afectan negativamente y le producen malestar. Diversos autores (Fierro, 1997; Muñoz et al., 1998, Lazarus \& Folkman, 1986; Lazarus, 2000) mencionan que los estímulos nuevos, impredecibles, incontrolables, ambiguos, inminentes, no deseables y de larga duración tienen mayor probabilidad de ser valorados como amenazantes.

En el año 2001, y a partir de este modelo teórico, se creó en Estados Unidos una escala para evaluar el estrés asociado a diferentes demandas y recursos propios del trabajo docente en escuelas primarias (Lambert et al., 2001). Entre las demandas se incluyeron los problemas de conducta y las inasistencias de los estudiantes, las exigencias administrativas, la escasez de material didáctico, etcétera. Entre los recursos, se evaluaron la disponibilidad y ayuda del personal de apoyo escolar, el acompañamiento administrativo, el respaldo de la comunidad y la capacitación especializada. Los investigadores identificaron el mayor riesgo de estrés ocupacional en aquellos docentes que percibían altas demandas y bajos recursos (Lambert et al., 2019).

En una línea similar, Salanova et al. (2003) observaron que las fuentes más importantes de estrés en docentes españoles de nivel secundario estuvieron asociadas a las siguientes demandas: la sobrecarga de trabajo (i.e. exceso de tareas y poco tiempo para realizarlas), la sobrecarga emocional (i.e. implicación afectiva en relaciones con alumnos, padres y sociedad), la ambigüedad de rol (i.e. no saber qué esperan la institución, los alumnos, los padres y la comunidad), el conflicto de rol (i.e. recibir instrucciones contradictorias en cuanto a los cometidos laborales), la falta de apoyo de los colegas, la falta de coordinación en el trabajo en equipo, la desmotivación e indisciplina de los alumnos, y los obstáculos técnicos (i.e. escasez de materiales, fallas en las instalaciones, etc.).

Por su parte, un análisis cualitativo sobre la base de 226 relatos proporcionados por docentes argentinos que enseñaban en los niveles inicial y primario, destacó que las demandas percibidas como estresantes se concentraban en torno a los siguientes tópicos: 


\section{Oros, VARgas Rubilar, \& CHEMISQUY}

los conflictos con superiores y colegas (e.g. injusticias, discusiones, maltratos), los problemas con los estudiantes (e.g. dificultades de comportamiento, inatención, accidentes, etc.), los problemas con las familias de los estudiantes (e.g. falta de apoyo, quejas, críticas, escaso acompañamiento al alumno), el descontento con el entorno físico de trabajo (e.g. ubicación, infraestructura, orden y limpieza), y las demandas propias del cargo (e.g. sobrecarga, conflicto de rol, incertidumbre sobre el futuro laboral). De todos ellos, los estresores asociados a los estudiantes y a los directivos y colegas, en ese orden, fueron los más prominentes (Menghi, 2015).

En el ámbito universitario, una investigación realizada con docentes mexicanos, reveló que las principales fuentes de estrés ocupacional tenían que ver con el grado de reconocimiento (e.g. inequidad y dificultades en los programas de estímulo) y con la sobrecarga de trabajo (e.g. multiplicidad de tareas, reuniones prolongadas, comisiones y actividades no planificadas), seguidas por los factores institucionales (e.g. liderazgo, autonomía, relación con colegas y superiores) y por las condiciones físicas del ambiente (e.g. limpieza, equipamiento) (Urquidi Treviño \& Rodríguez Jiménez, 2010).

Cuando los docentes universitarios ejercen su función en programas de Educación a Distancia, se suman además los estresores propios del uso de las nuevas tecnologías. Así lo evidenció un estudio realizado con profesores y tutores brasileños quienes informaron no sólo sentirse especialmente afectados por el conflicto y la ambigüedad de rol, la conciliación familia-trabajo y las presiones laborales, sino también por el uso sostenido de tecnologías de la información (Goebel \& Carlotto, 2019).

Resumiendo estos aportes, se puede concluir que los estresores laborales del ámbito docente provienen de al menos cuatro fuentes principales, tal como lo expone Peiró (2005). Este autor, con el fin de presentar y describir desencadenantes del estrés en el ámbito laboral, clasifica los estresores en cuatro categorías. En primer lugar se destacan el ambiente físico (e.g., ruido, temperatura, iluminación, humedad, higiene, ventilación, disponibilidad de espacio físico para desempeñar el trabajo, etc.), las características de las tareas que hay que realizar (e.g., la oportunidad de control, el uso de habilidades que permite la realización de las tareas, el feedback que se recibe por la propia ejecución del trabajo, la identidad de la tarea y la complejidad del propio trabajo) y las demandas del trabajo (e.g., sobrecarga laboral, el turno de trabajo, exposición a riesgos, etc.). A continuación se resaltan el desempeño del rol (e.i., el conflicto, la ambigüiedad y la sobrecarga de rol), las relaciones interpersonales en el trabajo (e.g., con superiores, con compañeros, subordinados, clientes, etc.) y el desarrollo de la carrera (e.g., inseguridad 
laboral, promociones excesivamente rápidas o las demasiado lentas, etc.) En tercer lugar se señalan el uso de las nuevas tecnologías (e.g., problemas de diseño, de implantación y de gestión de las mismas) y otros aspectos organizacionales (e.g., estructura o clima organizacional). Por último, se destacan estresores laborales que tienen que ver con las relaciones entre el trabajo y otras esferas de la vida, como pueden ser las demandas conflictivas entre la actividad laboral y los roles familiares.

Las condiciones de aislamiento y distanciamiento social en las que actualmente los docentes se ven obligados a desarrollar su actividad podrían dotar a estos potenciales estresores de una mayor valencia negativa; además, debido a su impacto acumulativo vinculado a otros estresores relacionados con las preocupaciones por la salud y el contagio, con la situación socioeconómica del país y con la pérdida de contacto físico con personas significativas, sentarían las bases para un malestar posiblemente más significativo. Un estudio realizado recientemente con 383 docentes mexicanos de bachillerato, licenciatura y posgrado, refiere que el $43.3 \%$ de los educadores que ejercen su labor durante la cuarentena obligatoria se sienten abrumados por cuestiones logísticas de su tarea; el 39.7\% refiere estrés por circunstancias relacionadas al uso de tecnologías; el 35.2\% menciona aspectos pedagógicos relacionados a la educación a distancia; y el 14.9\% señala situaciones sociales, afectivas y de salud (Sánchez Mendiola et al., 2020).

Si éstas y otras situaciones relacionadas al ámbito laboral son percibidas como amenazantes, y los recursos de afrontamiento para hacerles frente resultan insuficientes, se dispara la respuesta de estrés que será proporcional al valor negativo que se haya atribuido a esos eventos. Las respuestas psicofísicas de estrés suelen ser muy variadas. Puntualmente en población docente, se han observado con frecuencia los siguientes indicadores: dolores lumbares, dificultades para conciliar o mantener el sueño, problemas de voz, sensación de poco tiempo para uno mismo, sentido de urgencia constante, cansancio, irritabilidad, dolores de cabeza, resfríos y gripes, dificultades de memoria y concentración, cambios en el apetito, fatiga generalizada, nerviosismo, pérdida de placer y compromiso, etc. (Collado et al., 2016; Extremera et al., 2010; Harmsen et al., 2019; Tacca Huamán \& Tacca Huamán, 2019). La experimentación de estas manifestaciones es variable y puede estar afectada por la intervención de variables externas y de aspectos demográficos, como el género y la edad (Tacca Huamán \& Tacca Huamán, 2019).

Un artículo publicado recientemente por Villafuerte Holguín et al. (2020), recomienda a los educadores prestar especial atención a los cambios que se presenten en los planos cognitivo, afectivo, somático, interpersonal y conductual durante el periodo de 


\section{Oros, VARGas Rubilar, \& ChemisquY}

cuarentena por el COVID-19 dado que pueden limitar significativamente su bienestar y el progreso normal de su trabajo. La relación entre el estrés y el estado de salud ha sido sobradamente documentada (Seijas-Solano, 2019; Lousinha \& Guarino, 2013), por lo que resulta esencial contar con dispositivos que permitan detectar a tiempo los factores desencadenantes de estrés y sus posibles consecuencias.

Atendiendo a estas consideraciones, el presente estudio tuvo como objetivo desarrollar un instrumento para detectar la percepción de estresores docentes en contexto de aislamiento social, y estudiar su funcionamiento psicométrico: poder discriminativo de los ítems, consistencia interna y validez de constructo (factorial y nomológica). Con relación a la validez nomológica, a partir del modelo teórico general de Lazarus y Folkman (1986) y de evidencias más recientes en población docente (e.g. Harmsen et al., 2019; Oramas Viera et al., 2007), se hipotetizó una correlación positiva y significativa entre los puntajes de percepción de estrés y los puntajes obtenidos con una escala que mide síntomas físicos y psicológicos.

Identificar los estresores laborales docentes y conocer en qué medida estas situaciones son percibidas como amenazantes por los educadores podría ser de gran relevancia para las autoridades educativas provinciales y nacionales. Principalmente porque esta información permitiría valorar las condiciones en las cuales los profesores y maestras se encuentran ejerciendo su trabajo durante la crisis sanitaria por el COVID-19. A partir de estos resultados se podrían revisar las líneas de acción que se vienen implementando para dar continuidad a los procesos educativos de manera no presencial, así como también se podrían diseñar y desarrollar diferentes estrategias que favorezcan el bienestar y el cuidado de la salud de los educadores.

\section{Método}

\section{Diseño y tipo de estudio}

Se realizó una investigación empírica, no experimental, cuantitativa y transversal; de tipo instrumental (Montero \& León, 2007).

\section{Participantes}

Para la fase de elaboración y análisis de la validez de contenido del cuestionario se seleccionaron intencionalmente 14 profesionales del área educativa: $64 \%$ eran mujeres y $36 \%$ varones; en cuanto a sus funciones, cuatro eran directivos, uno era docente de nivel 
inicial, dos eran docentes de nivel primario, dos eran docentes de nivel medio y cinco eran docentes de nivel superior. Estos ejercían su función en establecimientos de gestión tanto pública $(n=4)$ como privada $(n=10)$, de las provincias de Buenos Aires $(n=2)$, Mendoza $(n=8)$, Entre Ríos $(n=1)$ y Misiones $(n=3)$.

Los demás análisis fueron ejecutados en una muestra de 674 docentes, de la cual aproximadamente el $40 \%$ fue utilizado con fines exploratorios, en tanto que el $60 \%$ restante se utilizó para el estudio confirmatorio. Así, para analizar el poder discriminativo de los ítems, la validez factorial exploratoria y la consistencia interna del instrumento, se trabajó con una submuestra de 289 docentes ( $75 \%$ mujeres; $M$ edad $=39,27 ; D E=9,64$ ), pertenecientes a diferentes niveles del sistema educativo, concertados en diversos sectores socioeconómicos de 17 provincias argentinas. Por otra parte, para poner a prueba el ajuste del modelo factorial, se trabajó con 385 docentes ( $78 \%$ mujeres; $M$ edad = 38,95; $D E=8,69)$ pertenecientes a todos los niveles educativos y a dos modalidades de la educación (i.e. Educación Especial y Educación Permanente de Jóvenes y Adultos), establecidos en diversos sectores socioeconómicos de 22 provincias argentinas. La descripción de otras características laborales de los participantes puede consultarse en la Tabla 1.

Para la selección de los participantes se empleó un procedimiento no probabilístico, por disponibilidad, de reclusión de voluntarios. El tamaño muestral para la validación del instrumento se consideró aceptable puesto que está recomendado entre 200 y 400 casos para garantizar la estabilidad y precisión de las soluciones factoriales (Kline, 2015; Lloret-Segura et al., 2014).

\section{Instrumentos}

\section{Escala de Estresores Docentes en Tiempos de Pandemia}

Para cumplir con el propósito de este estudio se elaboró una escala, que en su versión final, quedó compuesta por 23 ítems escalados al estilo Likert. En los siguientes apartados se detallará su procedimiento de construcción y sus resultantes propiedades psicométricas. 
Tabla 1.

Características demográficas y laborales de los participantes

\begin{tabular}{|c|c|c|c|c|c|c|}
\hline \multirow[b]{2}{*}{ Variables } & \multicolumn{3}{|c|}{$\begin{array}{l}\text { Submuestra } 1 \\
\quad(\mathrm{n}=289)\end{array}$} & \multicolumn{3}{|c|}{$\begin{array}{l}\text { Submuestra } 2 \\
\quad(\mathrm{n}=385)\end{array}$} \\
\hline & $\%$ & $\mathrm{M}$ & $\mathrm{DE}$ & $\%$ & $\mathrm{M}$ & $\mathrm{DE}$ \\
\hline \multicolumn{7}{|l|}{ Género } \\
\hline Femenino & 75.4 & & & 78.4 & & \\
\hline Masculino & 24.6 & & & 21.6 & & \\
\hline Edad & & 39.27 & 9.64 & & 38.95 & 8.68 \\
\hline \multicolumn{7}{|l|}{ Nivel educativo/modalidad } \\
\hline Educación Inicial & 12.8 & & & 8.6 & & \\
\hline Educación Primaria & 43.6 & & & 31.9 & & \\
\hline Educación Secundaria & 43.3 & & & 40.3 & & \\
\hline Educación Superior & 0.3 & & & 15.1 & & \\
\hline Educación Especial & & & & 03.6 & & \\
\hline Educación Permanente de Jóvenes y Adultos & & & & 0.5 & & \\
\hline Antigüedad en la docencia & & 11.01 & 8.22 & & 11.14 & 7.51 \\
\hline \multicolumn{7}{|l|}{ Gestión de la institución educativa } \\
\hline Estatal & & & & 51.2 & & \\
\hline Privada & 100 & & & 36.4 & & \\
\hline Simultánea (gestión pública y privada) & & & & 12.4 & & \\
\hline \multicolumn{7}{|l|}{ Cantidad de alumnos a cargo } \\
\hline 1 a 25 alumnos & 20.1 & & & 16.4 & & \\
\hline 26 a 50 alumnos & 24.0 & & & 20.0 & & \\
\hline 51 a 100 alumnos & 15.6 & & & 19.0 & & \\
\hline 101 a 200 alumnos & 19.8 & & & 22.0 & & \\
\hline más de 201 alumnos & 20.5 & & & 22.6 & & \\
\hline Cantidad de horas asignadas & & 23.16 & 15.28 & & 23.20 & 14.81 \\
\hline
\end{tabular}

\section{Escala de Indicadores Físicos y Psicoemocionales de Estrés}

Para conocer los síntomas de estrés se administró la Escala de indicadores físicos y psicoemocionales de estrés (Oros \& Neifert, 2006). La misma consta de 22 ítems y permite obtener un puntaje general de sintomatología asociada a aspectos cognitivos (e.g., "Me cuesta concentrarme"; "Me olvido fácilmente de las cosas"), físicos (e.g.,"Tengo insomnio o dificultad para conciliar el sueño"; "Tengo dolor de cuello y espalda"), y nerviosismo (e.g., "Me siento muy nervioso por pequeñeces"; "Siento que me preocupo excesivamente por cada cosa”). Los ítems son respondidos en una escala Likert con cinco opciones de respuesta (Nunca, Rara vez, A veces sí - A veces no, Frecuentemente, Siempre). El valor general de alpha de Cronbach informado por las autoras fue de .86 (Oros \& Neifert, 2006), en tanto que en este trabajo se obtuvo un alpha de .83. 


\section{Procedimiento de construcción de la Escala de estresores docentes en tiempos de pandemia}

De los 14 docentes que participaron en la fase de fase de elaboración y análisis de la validez de contenido, tres actuaron como informantes clave aportando ideas para la identificación de situaciones laborales potencialmente amenazantes en contexto de aislamiento. Estos aportes fueron aunados con la bibliografía consultada (i.e. Lambert, et al., 2019; Menghi, 2015; Peiró, 2005; Sánchez Mendiola et. al., 2020; Tacca Huamán \& Tacca Huamán, 2019) dando lugar a la elaboración de 21 ítems, los cuales fueron escalados al estilo Likert, con cinco opciones de respuesta según el grado en que cada situación resultara estresante (Nada estresante $=1$, Poco estresante $=2$, Algo estresante $=3$, Bastante estresante $=4$, Muy estresante $=5$ ). Otros 11 docentes actuaron como jueces para evaluar cualitativamente la claridad de los reactivos, su pertinencia y su grado de ajuste a la realidad actual de los educadores. Asimismo, debieron juzgar si la escala evidenciaba aspectos redundantes u omisiones significativas. Las contribuciones de los jueces indicaron la necesidad de desdoblar un ítem y generar uno nuevo, dando lugar a una versión final de 23 reactivos.

\section{Procedimientos de recolección de datos}

Dadas las particulares condiciones de aislamiento social, preventivo y obligatorio en las que se desarrolló este proyecto, la Escala de Estresores Docentes en Tiempos de Pandemia se aplicó por medio de un formulario diseñado para ser administrado en modalidad on-line. La reclusión de voluntarios se realizó a través de redes sociales, correo electrónico y servicios de mensajería digital, en algunos casos con apoyo de autoridades institucionales. El tiempo estimado de respuesta por cada persona fue de cinco minutos. La recolección de los datos comenzó 46 días después de iniciado el aislamiento social preventivo en nuestro país, en un período comprendido entre el 27 de abril y el 20 de mayo de 2020. Los participantes debieron afirmar su consentimiento para participar voluntariamente del estudio. Se garantizó a los respondentes un manejo confidencial de sus datos, se les proporcionó una dirección de correo electrónico para eventuales consultas, y un espacio optativo al final de la plantilla de respuestas para expresar comentarios. 


\section{Oros, VARGas Rubilar, \& ChemisquY}

\section{Procedimientos de análisis de datos}

Las respuestas aportadas por los docentes fueron automáticamente registradas en una base de datos compatible con el programa estadístico para Ciencias Sociales (SPSS v.19.0), en su versión para Windows.

Para describir la distribución de las puntuaciones se calcularon los indicadores de asimetría y curtosis univariada, para los cuales son deseables valores entre $+/-2$ (Tabachnick \& Fidell, 2013). Como complemento para estimar la normalidad univariada, se aplicó además la prueba de Kolmogorov-Smirnov y se analizaron los gráficos $Q-Q$ normal. La normalidad multivariada se testeó a través del coeficiente de Mardia, estableciéndose como apropiado un valor por debajo de 70 (Rodríguez \& Ruiz, 2008). El análisis del poder discriminativo de los reactivos se estudió mediante la correlación ítemtotal corregida, para la cual son esperables valores de $r$ iguales o superiores a .30 (Nunnally \& Bernstein, 1995).

La adecuación muestral de datos se analizó mediante el test de esfericidad de Bartlett y el índice de Kaiser-Meyer-Okin (KMO) (Carretero-Dios \& Pérez, 2005). El Análisis Factorial Exploratorio (AFE) se llevó a cabo con el método de Ejes principales y rotación Oblimín directo, dado que no se disponía de argumentos para hipotetizar independencia entre los posibles factores. Para la extracción de estos últimos se consideraron el criterio de autovalores superiores a 1, el gráfico de sedimentación de Cattell, el Análisis Paralelo (ejecutado con el programa Factor; Lorenzo-Seva \& Ferrando, 2006) y la interpretabilidad de la estructura factorial observada.

Para decidir la retención de los ítems y factores se tomaron en consideración las siguientes recomendaciones: que las saturaciones de los ítems fueran superiores a |.30|, que la matriz resultara factorialmente simple, que hubiera al menos 3 ítems por factor, y que existiera significancia teórica entre el ítem y el factor (Lloret-Segura et al., 2014). El análisis de la consistencia interna fue realizado a través del coeficiente Omega de McDonald (Ventura-León \& Caycho-Rodríguez, 2017), para el cual se consideran adecuados los valores iguales o superiores a .70 .

Para el Análisis Factorial Confirmatorio se utilizó el software estadístico LISREL 10.20. El método empleado fue el de mínimos cuadrados ponderados diagonal (DWLS), y debido al carácter ordinal de los datos, se introdujo la matriz de correlaciones asintóticas. Para evaluar los resultados se tuvieron en cuenta los puntos de corte y criterios más habituales en la práctica tanto para las medidas de ajuste $(\chi 2 / \mathrm{gl}<2$; CFI, GFI y NNFI 
$\geq .95$ ) como para los índices de error (RMSEA $\leq .06$; SRMR $<1$ ) (Hooper et al., 2008; Tabachnick \& Fidell, 2013). La variancia común entre los elementos y su factor latente fue analizada mediante la Variancia Media Extractada (VME), la cual idealmente debe ser mayor a .50 (Hair et al., 2009).

Para estudiar la validez nomológica se utilizó la correlación $r$ de Pearson, tomando como indicadores del tamaño del efecto los siguientes valores: pequeño $(r<.30)$, moderado ( $r$ entre .30 y .50$)$ y grande $(r>.50)$.

\section{Consideraciones éticas}

Las acciones realizadas en el marco de este trabajo acataron las recomendaciones éticas internacionales para la investigación con seres humanos (i.e. American Psychological Association, 2017; Declaración de Helsinki, 2013). Los docentes participaron de forma anónima y voluntaria, dejando asentado su consentimiento antes de responder las consignas del formulario. No se brindaron incentivos de ningún tipo a cambio de la participación. La información recabada fue tratada confidencialmente, y no tuvieron acceso a ella personas ajenas a la investigación.

\section{Resultados}

\section{Análisis preliminares: Estadísticos descriptivos y poder discriminativo de los ítems}

Los indicadores de asimetría y curtosis resultaron apropiados, siendo en ambos casos y para todos los ítems, menores a +/- 1.50. Las pruebas de Kolmogorov-Smirnov mostraron niveles críticos por debajo de .05 , pero debido a que este estadístico es muy sensible a pequeñas desviaciones de la normalidad en muestras amplias, se siguió la recomendación de Pardo Merino y Ruiz Díaz (2005) de acompañar estos resultados con la observación de diagramas de normalidad. En todos los casos, los gráficos $Q$ - $Q$ normal mostraron un ajuste aceptable de los puntos a la diagonal, dando cuenta de una distribución que se aproxima a la normal. Por otra parte, con referencia al poder discriminativo de los ítems, las correlaciones ítem-total corregidas resultaron satisfactorias, oscilando entre .49 y .70 (véase Tabla 2). 
Oros, VARgas Rubilar, \& ChemisquY

Tabla 2.

Resultados de asimetría, curtosis y correlaciones ítem-total corregida

\begin{tabular}{|c|c|c|c|}
\hline Ítems & Asimetría & Curtosis & $\begin{array}{l}r \text { ítem-total } \\
\text { corregida }\end{array}$ \\
\hline $\begin{array}{l}\text { 1. Tener que modificar los contenidos y actividades para } \\
\text { adaptarlos a la modalidad virtual }\end{array}$ & -0.24 & -0.65 & .60 \\
\hline $\begin{array}{l}\text { 2. Recibir múltiples y simultáneas consultas de parte de los } \\
\text { alumnos y/o padres }\end{array}$ & -0.18 & -1.06 & 60 \\
\hline 3. Recibir quejas y críticas de padres y/o alumnos & 0.36 & -1.12 & .51 \\
\hline 4. Trabajar en un lugar donde es difícil concentrarse & 0.02 & -1.23 & .55 \\
\hline $\begin{array}{l}\text { 5. Recibir un mayor número de requerimientos y } \\
\text { exigencias por parte mis superiores }\end{array}$ & -0.60 & -0.73 & .65 \\
\hline $\begin{array}{l}\text { 6. Aprender a utilizar y dominar nuevos medios } \\
\text { tecnológicos }\end{array}$ & 0.04 & -1.16 & .57 \\
\hline 7. Estar físicamente alejada del resto del equipo docente & 0.14 & -1.00 & .49 \\
\hline $\begin{array}{l}\text { 8. Disponer de recursos tecnológicos insuficientes o } \\
\text { inadecuados (conexión a internet, computadora, celular, } \\
\text { etc.) }\end{array}$ & 0.06 & -1.35 & .59 \\
\hline $\begin{array}{l}\text { 9. Tener poco tiempo para realizar todas las tareas que } \\
\text { implica el trabajo a distancia }\end{array}$ & -0.47 & -0.99 & .70 \\
\hline $\begin{array}{l}\text { 10. Ocuparme de situaciones que le competen a la familia } \\
\text { del alumno o a otros actores (trabajadores sociales, } \\
\text { psicólogos, psicopedagogos, etc.) }\end{array}$ & 0.13 & -1.27 & .62 \\
\hline $\begin{array}{l}\text { 11. Tener poca participación en la selección de plataformas } \\
\text { virtuales }\end{array}$ & 0.21 & -1.16 & .58 \\
\hline 12. Mantener mi propia motivación para seguir adelante & 0.15 & -1.18 & .64 \\
\hline $\begin{array}{l}\text { 13. Trabajar sin un horario ordenado, predeterminado o } \\
\text { diferente al que cumplía habitualmente }\end{array}$ & -0.55 & -0.10 & .64 \\
\hline $\begin{array}{l}\text { 14. Ocuparme simultáneamente de mi trabajo y de las tareas } \\
\text { del hogar (cocinar, limpiar, etc.). }\end{array}$ & -0.69 & -0.74 & .62 \\
\hline $\begin{array}{l}\text { 15. Notar que a pesar del esfuerzo que realizo mis alumnos } \\
\text { no progresan o no están motivados }\end{array}$ & -0.12 & -1.05 & .61 \\
\hline $\begin{array}{l}\text { 16. Recibir presiones de mi entorno familiar para que } \\
\text { trabaje menos horas }\end{array}$ & 0.26 & -1.33 & .57 \\
\hline $\begin{array}{l}\text { 17. Sentir que los superiores no comprenden lo difícil que } \\
\text { es trabajar en estas condiciones }\end{array}$ & -0.10 & -1.41 & .63 \\
\hline $\begin{array}{l}\text { 18. Desconocer si realmente los estudiantes están } \\
\text { comprendiendo los contenidos }\end{array}$ & -0.43 & -0.69 & .59 \\
\hline $\begin{array}{l}\text { 19. Tener poco conocimiento y manejo de programas y } \\
\text { aplicaciones que puedan favorecer la educación a } \\
\text { distancia }\end{array}$ & 0.30 & -1.13 & .55 \\
\hline $\begin{array}{l}\text { 20. Saber que hay alumnos que no cuentan con los recursos } \\
\text { tecnológicos para trabajar virtualmente }\end{array}$ & -0.63 & -0.58 & .55 \\
\hline $\begin{array}{l}\text { 21. Tener incertidumbre hacia el futuro, no saber cuándo va } \\
\text { a terminar la pandemia }\end{array}$ & -0.43 & -1.13 & .54 \\
\hline $\begin{array}{l}\text { 22. A partir de esta situación desconocer cómo seguirá mi } \\
\text { situación socioeconómica }\end{array}$ & -0.28 & -1.27 & .55 \\
\hline $\begin{array}{l}\text { 23. Tener poca participación en la elección de vías de } \\
\text { comunicación, criterios de evaluación, etc. }\end{array}$ & -0.02 & -1.30 & .63 \\
\hline
\end{tabular}




\section{Análisis factorial exploratorio y consistencia interna}

Los indicadores de adecuación muestral habilitaron la realización del AFE (KMO $=.92$; Chi cuadrado de Bartlett $=3490.86 ; \mathrm{p}<.001)$. El gráfico de sedimentación de Cattell y el Análisis Paralelo sugirieron una estructura unidimensional de los datos, en tanto que el criterio de autovalores mayores a 1 apuntó a una solución de 5 factores, por lo que se definió explorar ambas opciones.

Las cargas factoriales de los 23 ítems en la solución unidimensional oscilaron entre $|.52|$ y $|.72|$, explicando el $39 \%$ de la varianza total. El valor de consistencia interna resultó excelente $(\omega=.92)$. (véase Tabla 3$)$.

Tabla 3.

Cargas factoriales de la solución unidimensional

\begin{tabular}{|c|c|}
\hline Ítems & $\begin{array}{l}\text { Factor } \\
\text { único }\end{array}$ \\
\hline 1. Tener que modificar los contenidos y actividades para adaptarlos a la modalidad virtual & .61 \\
\hline 2. Recibir múltiples y simultáneas consultas de parte de los alumnos y/o padres & .61 \\
\hline 3. Recibir quejas y críticas de padres y/o alumnos & .52 \\
\hline 4. Trabajar en un lugar donde es difícil concentrarse & .63 \\
\hline 5. Recibir un mayor número de requerimientos y exigencias por parte mis superiores & 67 \\
\hline 6. Aprender a utilizar y dominar nuevos medios tecnológicos & .63 \\
\hline 7. Estar físicamente alejada del resto del equipo docente & .53 \\
\hline $\begin{array}{l}\text { 8. Disponer de recursos tecnológicos insuficientes o inadecuados (conexión a internet, } \\
\text { computadora, celular, etc.) }\end{array}$ & .63 \\
\hline 9. Tener poco tiempo para realizar todas las tareas que implica el trabajo a distancia & .71 \\
\hline $\begin{array}{l}\text { 10. Ocuparme de situaciones que le competen a la familia del alumno o a otros actores } \\
\text { (trabajadores sociales, psicólogos, psicopedagogos, etc.) }\end{array}$ & .67 \\
\hline 11. Tener poca participación en la selección de plataformas virtuales & .56 \\
\hline 12. Mantener mi propia motivación para seguir adelante & 60 \\
\hline $\begin{array}{l}\text { 13. Trabajar sin un horario ordenado, predeterminado o diferente al que cumplía } \\
\text { habitualmente }\end{array}$ & .66 \\
\hline $\begin{array}{l}\text { 14. Ocuparme simultáneamente de mi trabajo y de las tareas del hogar (cocinar, limpiar, } \\
\text { etc.). }\end{array}$ & .72 \\
\hline $\begin{array}{l}\text { 15. Notar que a pesar del esfuerzo que realizo mis alumnos no progresan o no están } \\
\text { motivados }\end{array}$ & .62 \\
\hline 16. Recibir presiones de mi entorno familiar para que trabaje menos horas & 60 \\
\hline 17. Sentir que los superiores no comprenden lo difícil que es trabajar en estas condiciones & .65 \\
\hline 18. Desconocer si realmente los estudiantes están comprendiendo los contenidos & .63 \\
\hline $\begin{array}{l}\text { 19. Tener poco conocimiento y manejo de programas y aplicaciones que puedan favorecer } \\
\text { la educación a distancia }\end{array}$ & .65 \\
\hline $\begin{array}{l}\text { 20. Saber que hay alumnos que no cuentan con los recursos tecnológicos para trabajar } \\
\text { virtualmente }\end{array}$ & 62 \\
\hline 21. Tener incertidumbre hacia el futuro, no saber cuándo va a terminar la pandemia & .59 \\
\hline 22. A partir de esta situación desconocer cómo seguirá mi situación socioeconómica & .57 \\
\hline $\begin{array}{l}\text { 23. Tener poca participación en la elección de vías de comunicación, criterios de } \\
\text { evaluación, etc. }\end{array}$ & .61 \\
\hline
\end{tabular}

Por su parte, la solución pentafactorial fue analizada a partir de las matrices de estructura y de configuración, resultando esta última más parsimoniosa. Las saturaciones factoriales oscilaron entre $|.31|$ y $|.90|$, excepto para los ítems 7 (e.i., "Estar físicamente 


\section{Oros, VARgas Rubilar, \& CHEMISQUY}

alejada/o del resto del equipo docente") y 12 (e.i., "Mantener mi propia motivación para seguir adelante") que presentaron cargas factoriales por debajo de |.30|, razón por la que quedaron descartados, dando como resultado una versión de 21 ítems.

Los cinco factores emergentes tienen sentido psicológico y evalúan estresores referidos al entorno de trabajo y la sobrecarga laboral (Factor 1), al empleo de nuevas tecnologías (Factor 2), a la incertidumbre por la duración y consecuencias de la pandemia para el docente y los alumnos (Factor 3), al aspecto organizacional de la institución educativa (Factor 4), y a las relaciones con el entorno del alumno, el conflicto y la ambigüedad de rol (Factor 5) (ver Tabla 4)

El ítem 5 (i.e., "Recibir un mayor número de requerimientos y exigencias por parte de mis superiores"), redactado originalmente para evaluar aspectos organizacionales de la institución (Factor 4), resultó factorialmente complejo, saturando también en el factor de Relaciones con el entorno del alumno, conflicto y ambigüedad de rol. Este resultado es comprensible dado que el contenido del ítem puede aportar tanto a una como a otra dimensión. La diferencia entre las cargas simultáneas fue insignificante desde el punto de vista métrico, por lo que hubiera sido recomendable eliminar o reformular el ítem. Sin embargo, dada la relevancia teórica que presenta el mismo, su contribución a la consistencia interna del Factor 4 (la consistencia interna disminuye si se elimina el elemento) y las limitaciones especiales de tiempo en las que se realizó esta investigación, se definió conservarlo en el factor con el que guardaba la mayor coherencia teórica (Factor 4) hasta probar el funcionamiento de la escala mediante el AFC.

Las correlaciones entre los factores resultaron entre moderadas $(r=.34)$ y altas $(r$ $=.55$ ), apoyando la elección de la rotación oblicua. Los valores de consistencia interna fueron satisfactorios para las cinco dimensiones (Factor 1: $\omega=.85$; Factor 2: $\omega=.85$; Factor 3: $\omega=.82$; Factor $4: \omega=.82$; Factor $5: \omega=.78$ ) y para la escala total de 21 ítems $(\omega=.93)$. 
Tabla 4.

Cargas factoriales para la matriz de configuración de cinco factores

\begin{tabular}{|c|c|c|c|c|c|c|}
\hline & \multirow{2}{*}{ Ítems } & \multicolumn{5}{|c|}{ Factores } \\
\hline & & 1 & 2 & 3 & 4 & 5 \\
\hline 9. & $\begin{array}{l}\text { Tener poco tiempo para realizar todas las tareas que implica } \\
\text { el trabajo a distancia }\end{array}$ & .47 & -.22 & -.13 & -.13 & .20 \\
\hline 13. & $\begin{array}{l}\text { Trabajar sin un horario ordenado, predeterminado o diferente } \\
\text { al que cumplía habitualmente }\end{array}$ & .51 & -.23 & .10 & .00 & -.01 \\
\hline 4. & Trabajar en un lugar donde es difícil concentrarse & .69 & -.04 & .11 & .09 & .04 \\
\hline 14. & $\begin{array}{l}\text { Ocuparme simultáneamente de mi trabajo y de las tareas del } \\
\text { hogar (cocinar, limpiar, etc.). }\end{array}$ & .73 & -.03 & .11 & -.01 & .01 \\
\hline 16. & $\begin{array}{l}\text { Recibir presiones de mi entorno familiar para que trabaje } \\
\text { menos horas }\end{array}$ & .70 & .13 & -.01 & -.13 & .03 \\
\hline 1. & $\begin{array}{l}\text { Tener que modificar los contenidos y actividades para } \\
\text { adaptarlos a la modalidad virtual }\end{array}$ & .21 & -.56 & .02 & -.02 & .01 \\
\hline 6. & Aprender a utilizar y dominar nuevos medios tecnológicos & -.03 & -.90 & -.03 & .00 & .07 \\
\hline 8. & $\begin{array}{l}\text { Disponer de recursos tecnológicos insuficientes o } \\
\text { inadecuados (conexión a internet, computadora, celular, etc.) }\end{array}$ & .16 & -.33 & .11 & -.14 & .10 \\
\hline 19. & $\begin{array}{l}\text { Tener poco conocimiento y manejo de programas y } \\
\text { aplicaciones que puedan favorecer la educación a distancia }\end{array}$ & -.08 & -.76 & .12 & -.09 & .05 \\
\hline 15. & $\begin{array}{l}\text { Notar que a pesar del esfuerzo que realizo mis alumnos no } \\
\text { progresan o no están motivados }\end{array}$ & .11 & .16 & .56 & -.07 & .28 \\
\hline 18. & $\begin{array}{l}\text { Desconocer si realmente los estudiantes están comprendiendo } \\
\text { los contenidos }\end{array}$ & .06 & -.04 & .65 & -.07 & .05 \\
\hline 20. & $\begin{array}{l}\text { Saber que hay alumnos que no cuentan con los recursos } \\
\text { tecnológicos para trabajar virtualmente }\end{array}$ & .11 & -.23 & .45 & -.04 & .02 \\
\hline 21. & $\begin{array}{l}\text { Tener incertidumbre hacia el futuro, no saber cuándo va a } \\
\text { terminar la pandemia }\end{array}$ & .06 & -.20 & .51 & -.04 & .01 \\
\hline 22. & $\begin{array}{l}\text { A partir de esta situación desconocer cómo seguirá mi } \\
\text { situación socioeconómica }\end{array}$ & .15 & -.01 & .38 & -.22 & .01 \\
\hline 7. & Estar físicamente alejada del resto del equipo docente & .03 & -.27 & .28 & -.05 & .01 \\
\hline 12. & Mantener mi propia motivación para seguir adelante & .20 & -.26 & .25 & -.14 & -.05 \\
\hline 5. & $\begin{array}{l}\text { Recibir un mayor número de requerimientos y exigencias por } \\
\text { parte mis superiores }\end{array}$ & .20 & -.18 & -.13 & -.31 & .32 \\
\hline 17. & $\begin{array}{l}\text { Sentir que los superiores no comprenden lo difícil que es } \\
\text { trabajar en estas condiciones }\end{array}$ & .17 & .04 & -.04 & -.52 & .25 \\
\hline 11. & $\begin{array}{l}\text { Tener poca participación en la selección de plataformas } \\
\text { virtuales }\end{array}$ & .06 & -.07 & .01 & -.69 & -.07 \\
\hline 23. & $\begin{array}{l}\text { Tener poca participación en la elección de vías de } \\
\text { comunicación, criterios de evaluación, etc. }\end{array}$ & -.12 & .00 & .13 & -.86 & -.00 \\
\hline 2. & $\begin{array}{l}\text { Recibir múltiples y simultáneas consultas de parte de los } \\
\text { alumnos y/o padres }\end{array}$ & .12 & -.13 & -.08 & -.00 & .70 \\
\hline 3. & Recibir quejas y críticas de padres y/o alumnos & -.07 & .00 & .18 & .05 & .71 \\
\hline 10. & $\begin{array}{l}\text { Ocuparme de situaciones que le competen a la familia del } \\
\text { alumno o a otros actores (trabajadores sociales, psicólogos, } \\
\text { psicopedagogos, etc.) }\end{array}$ & .12 & -.01 & .04 & -.28 & .46 \\
\hline
\end{tabular}

Nota: Los valores absolutos superiores a .30 se muestran en negrita

\section{Análisis factorial confirmatorio y nuevas evidencias de consistencia interna}

A partir de estos resultados, y habiendo comprobado la normalidad multivariada de los datos (Mardia $=68.93$ ), se pusieron a prueba tres modelos mediante AFC: (a) un modelo unifactorial de 23 ítems (M1); (b) un modelo pentafactorial de 21 ítems (M2); (c) y una reespecificación del modelo anterior, con errores correlacionados sugeridos por los 
índices de modificación (ítems 4 y 16; 13 y 14; 6 y 19; 21 y 22; 5 y 17; 11 y 23 (M3). Como se puede observar en la Tabla 5, el M3 presentó las mejores bondades de ajuste, a pesar de que el RMSEA superó los límites sugeridos.

Tabla 5.

Índices de ajuste y error para los tres modelos propuestos

\begin{tabular}{cccccccc}
\hline Modelo & $\chi 2 / \mathrm{gl}$ & CFI & GFI & NNFI & RMSEA & 90\% CI RMSEA & SRMR \\
\hline M1 & 4.69 & .85 & .96 & .83 & .14 & {$[.14-.15]$} & .09 \\
M2 & 3.42 & .92 & .98 & .90 & .12 & {$[.12-.13]$} & .07 \\
M3 & 2.32 & .95 & .99 & .95 & .09 & {$[.09-.10]$} & .05 \\
\hline
\end{tabular}

El ítem 5 que había resultado complejo en la submuestra exploratoria, y que bajo el criterio de interpretabilidad fue incluido en el factor con el cual guardaba la mayor coherencia teórica, mostró en la submuestra confirmatoria una carga adecuada con dicho factor $(|.76|)$, por lo cual se determinó su conservación. Las correlaciones entre las variables latentes fueron elevadas, entre .63 y .83. Por otro lado, las cargas factoriales del M3 se hallaron entre .54 y .87 para el primer factor, entre .51 y .76 para el segundo, entre .67 y .81 para el tercero, entre .72 y .82 para el cuarto, y entre .67 y .82 para el quinto factor (ver Figura 1). Los valores $t$ superaron a 1.96 lo que indica que todas las cargas factoriales resultaron significativas. Por su parte, la Varianza Media Extractada (VME) fue aceptable para cuatro de los cinco factores (VME $=.52 ; .42 ; .55 ; .76$ y .56 para los factores 1 al 5, respectivamente).

Debido a la presencia de errores correlacionados, para verificar la consistencia interna se empleó una corrección del coeficiente omega de McDonald que permite atenuar este efecto (Ventura-León et al., 2018). Los resultados fueron discretos para el factor 2 y entre aceptables y buenos para las demás dimensiones $(\omega=.81 ; .67 ; .83 ; .80 ;$ y .79 para los factores del 1 al 5, respectivamente). La consistencia interna de la escala completa resultó excelente $(\omega=.95)$. 

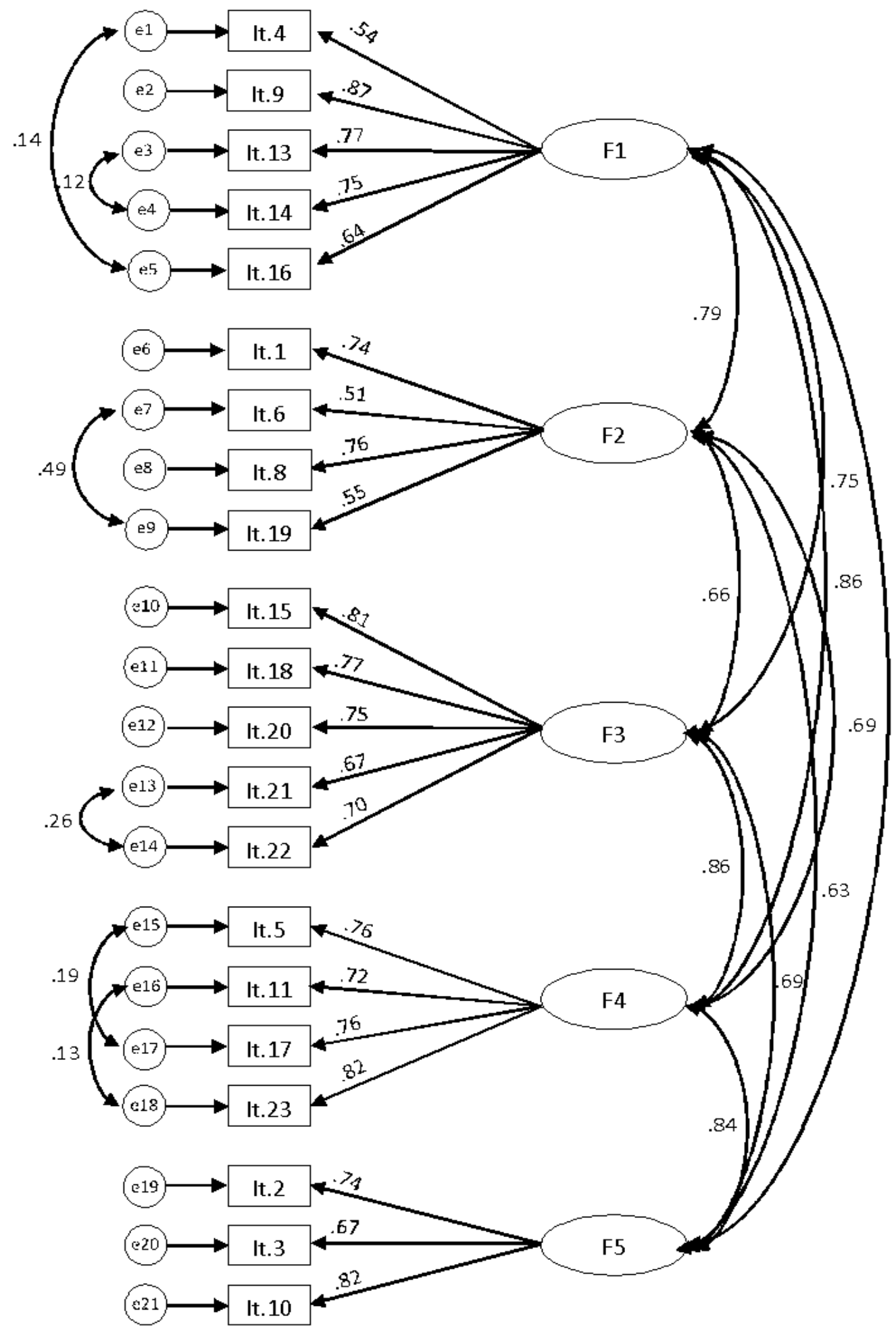

Figura 1. Modelo de 5 factores con errores correlacionados

En síntesis, la escala de estresores docentes en tiempos de pandemia quedó compuesta por 5 factores y 21 ítems. En la Tabla 6 se exponen los ítems que deben 


\section{Oros, VARGas Rubilar, \& CHEMISQUY}

sumarse para calcular el valor de estrés referido a cada factor, los valores mínimos y máximos posibles de obtener en cada una de estas dimensiones y en la escala total, así como los valores promedios y desviaciones típicas correspondientes a la muestra general de 674 docentes. Estos últimos valores podrían servir como referencia inicial para la interpretación de las puntuaciones en próximos estudios, hasta tanto no se disponga de un baremo.

Tabla 6.

Cálculo, puntajes mínimos y máximos, medias y desvíos para la Escala de Percepción de Estresores Docentes en Tiempos de Pandemia

\begin{tabular}{|c|c|c|c|c|}
\hline Factor/Escala & $\begin{array}{l}\text { Puntaje Mín. } \\
\text { posible }\end{array}$ & $\begin{array}{l}\text { Puntaje Máx. } \\
\text { posible }\end{array}$ & Media & Desvío \\
\hline $\begin{array}{c}\text { Factor } 1 \\
\text { (ítems } 4+9+13+14+16)\end{array}$ & 5 & 25 & 15.92 & 5.33 \\
\hline $\begin{array}{c}\text { Factor } 2 \\
\text { (items } 1+6+8+19)\end{array}$ & 4 & 20 & 12.07 & 4.19 \\
\hline $\begin{array}{c}\text { Factor } 3 \\
\text { (ítems } 15+18+20+21+22)\end{array}$ & 5 & 25 & 16.19 & 4.92 \\
\hline $\begin{array}{c}\text { Factor } 4 \\
\text { (ítems } 5+11+17+23)\end{array}$ & 4 & 20 & 11.94 & 4.51 \\
\hline $\begin{array}{c}\text { Factor } 5 \\
\text { (items } 2+3+10)\end{array}$ & 3 & 15 & 8.65 & 3.34 \\
\hline Escala total & 21 & 105 & 65.25 & 18.08 \\
\hline
\end{tabular}

\section{Análisis de la validez nomológica}

Con respecto al estudio de la validez nomológica, se observó una correlación positiva, significativa y de tamaño grande entre la puntuación general de percepción de estresores y los síntomas de estrés $(r=.53 ; p<.001)$. Las correlaciones obtenidas con cada una de las situaciones percibidas como estresantes también resultaron significativas, pero de menor tamaño: Entorno de trabajo y sobrecarga laboral $(r=.50 ; p<.001)$, Uso de nuevas tecnologías $(r=.40 ; p<.001)$, Incertidumbre por la duración y consecuencias de la pandemia $(r=.42 ; p<.001)$, Aspecto organizacional de la institución educativa $(r$ $=.43 ; p<.001), \mathrm{y}$ Relaciones con el entorno del alumno, conflicto $\mathrm{y}$ ambigüedad de rol $(r=.34 ; p<.001)$. 


\section{Discusión y Conclusión}

El objetivo de este estudio fue diseñar un instrumento para evaluar la percepción de estresores docentes en contexto de aislamiento social, y estudiar su funcionamiento psicométrico. Los análisis descriptivos dieron cuenta de una distribución de datos que se aproximó a la normalidad univariada, con valores de asimetría y curtosis adecuados (Bollen \& Long, 1993). Asimismo, indicaron correlaciones ítem-total corregidas por encima de los límites habitualmente sugeridos (Nunnally \& Bernstein, 1995), denotando un adecuado poder discriminativo de los ítems.

Los índices de adecuación muestral KMO y Chi cuadrado de Bartlett resultaron excelentes (el primero estuvo próximo a 1, y el segundo exhibió una muy baja probabilidad asociada al error de tipo I). El AFE indicó que tanto la solución unifactorial como la pentafactorial resultaban viables para explicar la distribución de los ítems, con valores de consistencia interna satisfactorios. Sin embargo, al poner a prueba ambas soluciones, el AFC reveló que el modelo unidimensional proveía un ajuste insuficiente a los datos. En cambio, el modelo de 5 factores mostró una mejor adecuación, la cual fue optimizada a partir de las correlaciones entre los errores de algunos elementos. Estos pares de elementos guardaban entre sí similitudes de contenido, lo cual puede explicar la variancia compartida. Atento a ello, y dado que la práctica de correlacionar errores puede restringir la generalización del modelo, se recomienda en futuros estudios prestar especial atención al comportamiento de estos ítems, y en caso de ser necesario, precisar mejor su redacción a fin de afirmar su especificidad.

El coeficiente omega corregido arrojó indicadores razonables de cohesión interna en el estudio confirmatorio. Si bien el factor 2 obtuvo un valor de consistencia por debajo de lo habitualmente recomendado (<.70), se encuentra bastante próximo al límite y puede ser tolerado debido al escaso número de ítems que lo componen (Loewenthal, 2001).

Por otra parte, aunque el $\chi 2 / g 1$ del M3 ostentó un valor ligeramente superior al que sugieren los criterios más exigentes, que señalan el límite por debajo de 2 (Tabachnick \& Fidell, 2013), se encuentra igualmente dentro de los parámetros aceptables (West et al., 2012). Asimismo, aunque el RMSEA superó el punto de corte inicialmente establecido $(<.06)$, se mantiene por debajo de .10, lo cual permite aun así considerarlo admisible (Browne \& Cudeck, 1992; MacCallum et al., 1996). De todos modos, los motivos de la inconsistencia observada entre el ajuste del CFI y el RMSEA debería ser profundizada en 


\section{Oros, VARGas Rubilar, \& ChemisquY}

próximos estudios ya que podría aportar información relevante acerca del ajuste del modelo propuesto (Lai \& Green, 2016).

Las correlaciones entre los factores fueron altas, denotando la existencia de algún posible solapamiento entre ellos (Gallagher \& Brown, 2013). La VME resultó aceptable para la mayoría de los factores, siendo más bien baja (.43 frente al .50 fijado como adecuado) para el factor 2: Uso de nuevas tecnologías. Esto sugiere que, en la presente muestra, la variancia de los ítems recogida por este factor está algo por debajo del $50 \%$ sugerido. En próximos estudios se recomienda comprobar nuevamente el comportamiento de este factor con la incorporación de un nuevo ítem, tal como se explicará a continuación.

A pesar de las etapas de revisión de expertos por las que pasó el instrumento, algunos docentes de la muestra final expresaron echar de menos la mención de un estresor significativo: "Tener que adquirir nuevos recursos tecnológicos y/o actualizar las prestaciones de telefonía e internet bajo un costo económico personal". Sería deseable en próximas aplicaciones de la escala incorporar este ítem para explorar su funcionamiento como parte del Factor 2: Uso de nuevas tecnologías. Esta adición podría modificar la consistencia interna del factor, que mostró un valor más bajo que el resto de las dimensiones en la muestra confirmatoria, y la variancia media extractada, cuyo valor estuvo por debajo de lo habitualmente esperable. En el Anexo de este artículo se puede consultar la versión definitiva de la escala con este elemento incorporado como ítem 22.

Por lo demás, y en términos generales, los estresores que sobresalen en la estructura pentafactorial armonizan con los hallazgos de estudios previos realizados en condiciones de no aislamiento. Si se comparan, por ejemplo, los cincos factores de la Escala de estresores docentes en tiempos de pandemia con la taxonomía propuesta por Peiró (2005) se aprecia que ambos modelos hacen referencia de manera similar a cuatro de los cinco estresores, aunque con algunas variaciones en la agrupación de los mismos. Por ejemplo, el modelo de Peiró incluye en una misma dimensión los estresores concernientes al uso de nuevas tecnologías y aquellos asociados a los aspectos organizacionales, mientras que en nuestro caso estos estresores forman parte de dimensiones independientes, tal como sucedió en el estudio de Goebel y Carlotto (2019). Por otra parte, y a diferencia de la propuesta de Peiró y de Ferreira et al. (2015), la dimensión entorno de trabajo y sobrecarga laboral se ha fusionado con la falta de conciliación trabajo-familia, lo cual es comprensible en este contexto de aislamiento y distanciamiento social. Durante los últimos meses, los docentes han tenido que realizar 
tareas laborales en su lugar de residencia, sin dejar de atender a responsabilidades de índole familiar y doméstico. Finalmente, en el presente trabajo emerge como un factor inédito la incertidumbre por la duración y consecuencias de la pandemia para el docente y los alumnos. Este factor reúne la sensación de amenaza que perciben los educadores ante una enfermedad hasta el momento desconocida, para la cual no existe aún una vacuna ni, por ende, una expectativa clara de su evolución y control. Desconocer la duración de las medidas de aislamiento y el impacto económico, social, educativo y sanitario de esta pandemia puede incrementar los sentimientos de desconcierto, preocupación y temor en estos profesionales, por lo cual su estudio merece ser profundizado en próximas investigaciones (Johnson et al., 2020).

Los resultados de la correlación entre la percepción de estresores y los indicadores psicofísicos de estrés están en línea con la teoría y con estudios precedentes (Lazarus \& Folkman, 1986; Harmsen et al., 2019), y replican con exactitud la magnitud de la correlación informada por Oramas et al. (2007). Los datos indican que los estresores que se asocian más fuertemente a la experiencia de síntomas indeseables devienen del entorno de trabajo y la sobrecarga laboral (Factor 1). Esto sugiere que los docentes que perciben un volumen de trabajo excesivo, que tienen poco tiempo para realizarlo y que además deben hacerlo en un lugar donde es difícil concentrarse, serían más susceptibles a experimentar nerviosismo, contracturas musculares, insomnio, dolores de cabeza, preocupaciones constantes, cambios de apetito, etc. Para futuros estudios se recomienda utilizar diseños predictivos o explicativos para estimar con precisión el impacto que estos estresores tienen sobre la salud y el bienestar de los educadores.

Como limitación general del estudio se puede remarcar el procedimiento de muestreo, ya que la selección de voluntarios puede introducir sesgos en las respuestas o en las interpretaciones de las mismas. Así, existe la posibilidad de que se hayan visto más motivados a participar de este estudio aquellos docentes que se identificaron con la temática por encontrarse laboralmente sobrepasados o, por el contrario, que hayan accedido a participar en mayor medida aquellos que no se encontraban sobreexigidos y por lo tanto disponían de más tiempo para formar parte de esta actividad. Sea como fuere, al tratarse de un muestreo no aleatorio, los resultados de esta investigación pueden no ser representativos del universo de estudio y por lo tanto tener una limitada capacidad de generalización (Sabino, 1996).

Otra limitación a destacar tiene que ver con el estudio de la fiabilidad de la escala, el cual se ha restringido únicamente el aspecto de la consistencia interna. Sería deseable 


\section{Oros, VARGas Rubilar, \& ChemisquY}

en próximas investigaciones analizar la estabilidad de las puntuaciones con medidas testre test.

Por último, esta investigación se limitó a estudiar la percepción de amenaza, sin evaluar los recursos de afrontamiento con que los docentes hacían frente a estas demandas, lo cual hubiera aportado una visión más completa y profunda del fenómeno, dado que según la teoría transaccional (Lazarus \& Folkman, 1986) es la combinación de ambos procesos lo que determina qué tan vulnerable al estrés es un individuo. Se sugiere abordar esta temática en próximas investigaciones, así como también el estudio de otros factores personales y sociales que podrían incidir sobre la percepción de amenazas docentes en este tiempo de contingencia: variables sociodemográficas y laborales, creencias personales y existenciales, rasgos de personalidad, etc.

Como conclusión general, y a pesar de las limitaciones destacadas, se puede afirmar que la escala de estresores docentes en tiempos de pandemia presenta adecuadas propiedades psicométricas y puede ser utilizada para continuar con el estudio de esta temática en tanto se prolongue la educación remota. Asimismo, también podrá ser capitalizada en futuras investigaciones post pandemia, en el marco de estudios retrospectivos.

Recoger información relativa al estrés laboral en docentes tiene gran relevancia, fundamentalmente porque diversas investigaciones han podido determinar que experimentar niveles moderados y altos de estrés conlleva consecuencias nocivas para el educador (e.g. repercute negativamente en la salud física como mental), para los destinatarios de los servicios educativos (e.g. afecta la calidad de la enseñanza ofrecida y debilita el vínculo pedagógico que se establece con los estudiantes) y para la institución escolar (e.g. incremento de las licencias médicas, el ausentismo laboral y el abandono de la profesión) (Holmes, 2020; Moriana Elvira \& Herruzo Cabrera, 2004; Ramberg et al., 2019). Los educadores han sido actores esenciales para dar continuidad y sostener los procesos educativos de manera no presencial durante los últimos meses. Por este motivo, resulta de vital importancia cuidar la salud y el bienestar de los profesionales de la educación hasta que se garantice el retorno seguro a los establecimientos educativos en todos los niveles y modalidades del Sistema Educativo. 


\section{Referencias}

American Psychological Association. (2017). Ethical Principles of Psychologists and Code of Conduct. https://www.apa.org/ethics/code

Asamblea Médica Mundial. (2013). Declaración de Helsinki de la AMM - Principios éticos para las investigaciones médicas en seres humanos. http://repositorio.mederi.com.co/bitstream/handle/123456789/386/Declaracio n-Helsinki-2013-Esp.pdf? sequence $=1$

Bollen, K. A., \& Long, J. S. (1993). Testing structural equation models. Newbury Park: Sage.

Browne, M. W., \& Cudeck, R. (1992). Alternative ways of assessing model fit. Sociological Methods \& Research, 21(2), 230-258. https://doi.org/10.1177/0049124192021002005

Carretero-Dios, H., \& Pérez, C. (2005). Normas para el desarrollo y revisión de estudios instrumentales. International Journal of Clinical and Health Psychology, 5(3), 521-551.

Carnese, F. (16 de mayo 2020). Los docentes piden ayuda psicológica en la pandemia. La Mañana Neuquén. https://www.Imneuquen.com/los-docentespiden-ayuda-psicologica-la-pandemia-n705934

Collado, P. A., Soria, C. B., Canafoglia, E., \& Collado, S. A. (2016). Condiciones de trabajo y salud en docentes universitarios y de enseñanza media de Mendoza, Argentina: Entre el compromiso y el desgaste emocional. Salud colectiva, 12, 203-220.

Di Vincenzo, D. (28 de marzo de 2020). Cuarentena docente: entre las mamaderas, las compras y las clases virtuales. Infobae.

https://www.infobae.com/historias/2020/03/28/cuarentena-docente-entre-lasmamaderas-las-compras-y-las-clases-virtuales/

Esteve, J. M. (1994). El malestar docente. Buenos Aires: Paidós.

Extremera, N., Rey, L., \& Pena, M. (2010). La docencia perjudica seriamente la salud. Análisis de los síntomas asociados al estrés docente. Boletín de psicología, 100 (1), 43-54.

Favant, B. (24 de Junio de 2020). El testimonio de docentes santafesinas: "Las aulas nunca estuvieron vacías". Diario Uno Santa Fe.

https://www.unosantafe.com.ar/santa-fe/el-testimonio-docentes-santafesinaslas-aulas-nunca-estuvieron-vacias-n2592992.html

Fernández, M. (26 de Abril de 2020). Los docentes, desbordados en medio de la pandemia: "Mi día a día se convirtió en un caos". Infobae. https://www.infobae.com/educacion/2020/04/26/los-docentes-desbordadosen-medio-de-la-pandemia-mi-dia-a-dia-se-convirtio-en-un-caos/

Ferreira, M. C., Milfont, T. L., Corrêa e Silva, A. P., Araújo Fernandes, H., Pacheco Almeida, S., Mendonça, H. (2015). Escala para Avaliação de Estressores Psicossociais no Contexto Laboral: Construção e Evidências de Validade. Psicologia: Reflexão e Crítica, 28(2), 340-349. http://dx.doi.org/10.1590/1678-7153.201528214

Fierro, A. (1997). Estrés, Afrontamiento y Adaptación. En M. I. Hombrados Mendieta (coord.), Estrés y Salud (pp. 11-37). Valencia: Promolibro.

Figueroa Díaz, A. (05 de mayo de 2020). Los docentes, la cuarentena y la sobrecarga de las clases a distancia. Página 12. https://www.pagina12.com.ar/263725los-docentes-la-cuarentena-y-la-sobrecarga-de-las-clases-a-d 
Gallagher, M. W., \& Brown, T. A. (2013). Introduction to confirmatory factor analysis and structural equation modeling. En T. Teo (Ed.) Handbook of quantitative methods for educational research (287-314). Brill Sense.

Gil-Monte, P. R. (2005). El Síndrome de Quemarse por el Trabajo. Una enfermedad laboral en la sociedad del bienestar. Madrid: Ediciones Pirámide.

Gil-Monte, P. R., \& Peiró, J. M. (1997). Desgaste psíquico en el trabajo: El síndrome de quemarse. Madrid: Síntesis.

Goebel, D. K., \& Carlotto, M. S. (2019). Preditores da autoavaliação da Saúde Geral em docentes de Educação a Distância. Revista Iberoamericana de Educación a Distancia, 22(1), 309-323. http://dx.doi.org/10.5944/ried.22.1.21885

Hair, J. F., Black, W. C., Babin, B. J., \& Anderson, R. E. (2009). Multivariate Data Analysis (7th ed.). New York: Pearson Prentice Hall.

Harmsen, R., Helms-Lorenz, M., Maulana, R., van Veen, K., \& van Veldhoven, M. (2019). Measuring general and specific stress causes and stress responses among beginning secondary school teachers in the Netherlands. International Journal of Research \& Method in Education, 42(1), 91-108. http://dx.doi.org/10.1080/1743727X.2018.1462313

Holmes, E. (2020). El bienestar de los docentes. Guía para controlar el estrés y sentirse bien personal y profesionalmente. Madrid: Narcea S.A. Ediciones

Hooper, D., Coughlan, J., \& Mullen, M. R. (2008). Structural equation modelling: guidelines for determining model fit. Electronic Journal of Bussines Research Methods, 6(1), 53-60.

Johnson, M. C., Saletti Cuesta, L., \& Tumas, N. (2020). Emociones, preocupaciones y reflexiones frente a la pandemia del COVID-19 en Argentina. Ciência \& Saúde Coletiva, 25, 2447-2456. https://doi.org/10.1590/1413$\underline{81232020256.1 .10472020}$

Kline, R. B. (2015). Principles and practice of structural equation modeling. New York: Guilford publications.

Lai, K., \& Green, S. B. (2016). The problem with having two watches: Assessment of fit when RMSEA and CFI disagree. Multivariate behavioral research, 51(2-3), 220-239. http://dx.doi.org/10.1080/00273171.2015.1134306

Lambert, R., Boyle, L., Fitchett, P., \& McCarthy, C. (2019). Risk for occupational stress among US kindergarten teachers. Journal of Applied Developmental Psychology, 61, 13-20.

Lambert, R. G., McCarthy, C. J., \& Abbott-Shim, M. (2001). Classroom Appraisal of Resources and Demands, school-age version. Atlanta: Head Start Quality Research Center.

Lazarus, R. S. (2000). Estrés y emoción. Manejo e implicaciones en nuestra salud. Bilbao: Desclée de Brouwer.

Lazarus, R. S., \& Folkman, S. (1986). Estrés y procesos cognitivos. Barcelona: Martínez Roca

Lloret-Segura, S., Ferreres-Traver, A., Hernández-Baeza, A., \& Tomás-Marco, I. (2014). El análisis factorial exploratorio de los ítems: una guía práctica, revisada y actualizada. Anales de Psicología, 30(3), 1151-1169. http://dx.doi.org/10.6018/analesps.30.3.199361

Lorenzo-Seva, U., \& Ferrando, P. J. (2006). FACTOR: A computer program to fit the exploratory factor analysis model. Behavior Research Methods, 38(1), 8891. http://dx.doi.org/10.3758/BF03192753 
Lousinha, A., \& Guarino, L. (2013). Diferencias individuales como moderadoras de la relación estrés-salud en docentes universitarios. Suma psicológica, 10(1), 73-84. http://dx.doi.org/10.18774/448x.2013.10.38

Loewenthal, K. M. (2001). An introduction to psychological tests and scales. London: Psychology Press.

MacCallum, R. C., Browne, M. W., \& Sugawara, H. M. (1996). Power analysis and determination of sample size for covariance structure modeling. Psychological Methods, 1, 130-149. http://dx.doi.org/10.1037/1082989X.1.2.130

Manassero, M. A., Vázquez, A., Ferrer, V. A., Fornés, J., \& Fernández, M. C. (2003). Estrés y burnout en la enseñanza. Palma de Mallorca: Ediciones UIB.

Menghi, M. S. (2015). Recursos psicosociales y manejo del estrés en docentes (Tesis doctoral inédita). Universidad Católica Argentina "Santa María de los Buenos Aires", Buenos Aires, Argentina.

Montero, I., \& León, O. G. (2007). A guide for naming research studies in Psychology. International Journal of clinical and Health psychology, 7(3), 847-862.

Moriana Elvira, J. A., \& Herruzo Cabrera, J. (2004). Estrés y burnout en profesores. Internacional Journal of Clinical and Health Psychology, 4(3), 591-621.

Muñoz, M. L., García, C., \& Guisado, A. B. (1998). Esquizofrenia. En M. A. Vallejo Pareja, Manual de Terapias de Conductas (Vol. II) (pp.761-814). Madrid: Dykinson.

Nunnally, J. C., \& Bernstein, I. J. (1995). Teoría psicométrica. Madrid: McGrawHill.

Oramas Viera, A., Almirall Hernandez, P., \& Fernández, I. (2007). Estrés laboral y el síndrome de burnout en docentes venezolanos. Salud de los Trabajadores, 15(2), 71-87.

Oros, L. B. \& Neifert, I. (2006). Construcción y validación de una escala para evaluar indicadores físicos y psicoemocionales de estrés. Evaluar, 6, 1-14.

Othman, Z., \& Sivasubramaniam, V. (2019). Depression, Anxiety, and Stress among Secondary School Teachers in Klang, Malaysia. International Medical Journal, 26(2), 71-74.

Pardo Merino, A., y Ruiz Díaz, M. A. (2005). Análisis de datos con SPSS 13 Base. Madrid: Mc-Graw Hill.

Peiró, J. M. (2005). Desencadenantes del estrés laboral. Madrid: Pirámide.

Rodríguez, M., \& Ruiz, M. (2008). Atenuación de la asimetría y de la curtosis de las puntuaciones observadas mediante transformaciones de variables: incidencia sobre la estructura factorial. Psicológica, 29(2), 205-227.

Ramberg, J., Låftman, S. B., Åkerstedt, T., \& Modin, B. M. (2019). Teacher stress and students' school well-being: The case of upper secondary schools in Stockholm. Scandinavian Journal of Educational Research. https://doi.org/10.1080/00313831.2019.1623308

Sabino, C. (1996). El proceso de investigación. Buenos Aires: Lumen-Humanitas.

Salanova, M., Llorens, S., \& García-Renedo, M. (2003). ¿Por qué se están quemando los profesores. Prevención, trabajo y salud, 28, 16-20.

Sánchez Mendiola, M., Martínez Hernández, A. M. D. P., Torres Carrasco, R., Agüero Servín, M., Hernández Romo, A. K., Benavidez Lara, M., Rendón Cazales, V. J. \& Jaimes Vergara, C. (2020). Retos educativos durante la pandemia de COVID-19: una encuesta a profesores de la UNAM. Revista Digital Universitaria, 21(3), 1-24. 
Santoro, S. (19 de abril de 2020). Coronavirus: la escuela en cuarentena. Página 12. https://www.pagina12.com.ar/260580-coronavirus-la-escuela-en-cuarentena

Schaufeli, W. B. (2003). Past performance and future perspectives of burnout research. Journal of Industrial Psychology, 29(4), 1-15. http://dx.doi.org/10.4102/sajip.v29i4.127

Schaufeli, W. B., \& Enzmann, D. (1998). The burnout companion to study and practice: A critical analysis. London: Taylor \& Francis.

Seijas-Solano, D. E. (2019). Riesgos psicosociales, estrés laboral y síndrome burnout en trabajadores universitarios de una escuela de bioanálisis. Revista de Salud Pública, 21(1), 102-108. http://dx.doi.org/10.15446/rsap.v21n1.71907

Tabachnick, B., \& Fidell, L. S (2013). Using Multivariate Statistics (6th Ed.). Boston, MA: Pearson.

Tacca Huamán, D. R., \& Tacca Huamán, A. L. (2019). Factores de riesgos psicosociales y estrés percibido en docentes universitarios. Propósitos y Representaciones, 7(3), 323-353.

Urquidi Treviño, L. E., \& Rodríguez Jiménez, J. R. (2010). Estrés en profesorado universitario mexicano. Revista Electrónica Actualidades Investigativas en Educación, 10(2), 1-21. http://dx.doi.org/10.15517/aie.v10i2.10111

Vallejos, S. (3 de mayo de 2020). Coronavirus en la Argentina. Jornadas interminables y mucho estrés, así es el día a día de los maestros a un mes y medio de la cuarentena. La Nación. https://www.lanacion.com.ar/sociedad/coronavirus-argentina-jornadasinterminables-mucho-estres-asi-nid2361011

Vandenberghe, R., \& Huberman, A. M. (1999). Understanding and preventing teacher burnout: A sourcebook of international research and practice. New York: Cambridge University Press.

Ventura-León, J. L., \& Caycho-Rodríguez, T. (2017). El coeficiente Omega: un método alternativo para la estimación de la confiabilidad. Revista Latinoamericana de Ciencias Sociales, Niñez y Juventud,15(1), 625-627.

Ventura-León, J., Jara-Avalos, S., Garcia-Pajuelo, C., \& Ortiz-Saenz, C. (2018). Validación de una escala de perfeccionismo en niños peruanos. Actualidades en Psicología, 32(124), 16-33.

Villafuerte Holguín, J. S., Bello Piguave, J. E., Pantaleón Cevallos, Y., \& Bermello Vidal, J. O. (2020). Rol de los docentes ante la crisis del COVID-19. Una mirada desde el enfoque humano. Revista Electrónica Formación y Calidad Educativa, 8(1), 134-150.

West, S. G., Taylor, A. B., \& Wu, W. (2012). Model fit and model selection in structural equation modeling. En R. Hoyle (Ed.). Handbook of structural equation modeling, 209-231. 


\section{ANEXO \\ Escala de Estresores Docentes en Tiempos de Pandemia}

Señale en qué medida le han resultado estresantes o desbordantes las siguientes situaciones durante la cuarentena

\begin{tabular}{|c|c|c|c|c|c|}
\hline Situaciones & $\begin{array}{l}\text { Nada } \\
\text { estresante }\end{array}$ & $\begin{array}{l}\text { Poco } \\
\text { estresante }\end{array}$ & $\begin{array}{c}\text { Algo } \\
\text { estresante }\end{array}$ & $\begin{array}{c}\text { Bastante } \\
\text { estresante }\end{array}$ & $\begin{array}{l}\text { Muy } \\
\text { estresante }\end{array}$ \\
\hline $\begin{array}{l}\text { 1. Tener que modificar los } \\
\text { contenidos y actividades para } \\
\text { adaptarlos a la modalidad virtual }\end{array}$ & & & & & \\
\hline $\begin{array}{l}\text { 2. Recibir múltiples y simultáneas } \\
\text { consultas de parte de los alumnos } \\
\text { y/o padres }\end{array}$ & & & & & \\
\hline $\begin{array}{l}\text { 3. Recibir quejas y críticas de } \\
\text { padres y/o alumnos }\end{array}$ & & & & & \\
\hline $\begin{array}{l}\text { 4. Trabajar en un lugar donde es } \\
\text { difícil concentrarse }\end{array}$ & & & & & \\
\hline $\begin{array}{l}\text { 5. Recibir un mayor número de } \\
\text { requerimientos y exigencias por } \\
\text { parte mis superiores }\end{array}$ & & & & & \\
\hline $\begin{array}{l}\text { 6. Aprender a utilizar y dominar } \\
\text { nuevos medios tecnológicos }\end{array}$ & & & & & \\
\hline $\begin{array}{l}\text { 7. Disponer de recursos } \\
\text { tecnológicos insuficientes o } \\
\text { inadecuados (conexión a internet, } \\
\text { computadora, celular, etc.) }\end{array}$ & & & & & \\
\hline $\begin{array}{l}\text { 8. Tener poco tiempo para } \\
\text { realizar todas las tareas que } \\
\text { implica el trabajo a distancia }\end{array}$ & & & & & \\
\hline $\begin{array}{l}\text { 9. Ocuparme de situaciones que } \\
\text { le competen a la familia del } \\
\text { alumno o a otros actores } \\
\text { (trabajadores sociales, } \\
\text { psicólogos, psicopedagogos, etc.) }\end{array}$ & & & & & \\
\hline $\begin{array}{l}\text { 10. Tener poca participación en la } \\
\text { selección de plataformas } \\
\text { virtuales }\end{array}$ & & & & & \\
\hline
\end{tabular}




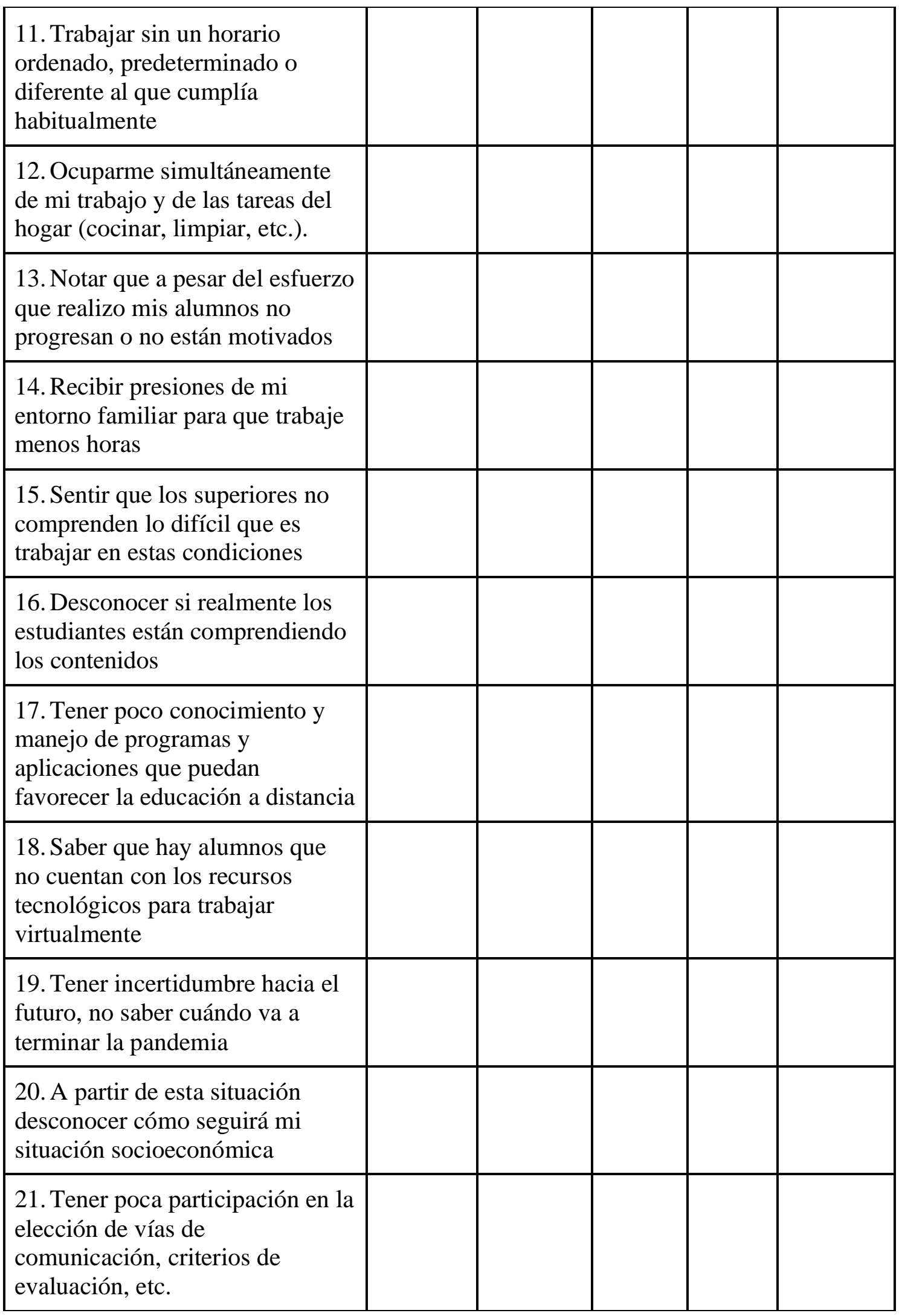


22. Tener que adquirir nuevos recursos tecnológicos y/o actualizar las prestaciones de telefonía e internet bajo un costo económico personal

\begin{tabular}{|l|l|l|l|l|}
\hline & & & & \\
& & & & \\
\hline
\end{tabular}

